\title{
Molecular diagnosis of Beckwith-Wiedemann Syndrome using quantitative methylation-sensitive polymerase chain reaction
}

Bradford Coffee, $P h D^{1}$, Kasinathan Muralidharan, $P h D^{1}$, William E. Highsmith Jr., PhD ${ }^{2}$, Pablo Lapunzina, $M D^{3}$, and Stephen T. Warren, $P h D^{1}$

\begin{abstract}
Purpose: Beckwith-Wiedemann Syndrome is caused by defects in imprinted gene expression at 11p15. Currently, quantitative Southern analysis using DNA methylation-sensitive restriction enzymes is used in molecular diagnosis of this syndrome. Methods: We describe a rapid and highly quantitative test for assessing DNA methylation at 11p15 using sodium bisulfite treatment of genomic DNA coupled with quantitative TaqMan methylation-sensitive polymerase chain reaction. Results: TaqMan MSP can assess DNA methylation at both differentially methylated region (DMR)1 and DMR2 at 11p15. In addition, by using TaqMan MSP we were able to determine the parent of origin of a duplication of 11p15 by quantification of both DMR1 and DMR2 DNA methylation. Conclusion: TaqMan MSP method is a robust and rapid method for detecting changes in DNA methylation that compares favorably to the current standard of Southern blot for DNA methylation analysis. Assessment of DMR1 and DMR2 provides the most comprehensive assay for methylation defects in Beckwith Wiedemann Syndrome, accounting for more than $70 \%$ of the cases. The advantages of TagMan MSP are that it requires less DNA and that it is rapid, less labor-intensive, and amenable to high-throughput analysis. Moreover, this approach can be modified to assess DNA methylation changes anywhere in the genome. Genet Med 2006:8(10):628-634.
\end{abstract}

Defects in imprinted gene expression at $11 \mathrm{p} 15$ are associated with Beckwith-Wiedemann Syndrome (BWS). ${ }^{1}$ More than 70\% of cases are found to have alterations in DNA methylation at two distinct differentially methylated regions (DMRs) at $11 \mathrm{p} 15 .^{2}$ DMR1 is located within the telomeric domain and controls the reciprocal imprinted expression of IGF2 and H19. The centromeric domain contains the KCNQ1, CDKN1C, SLC22A1L, and TSSC3 genes. The imprinted expression of these genes is controlled by KCNQ10T1, located on the antisense strand in intron 10 of the KCNQ1 gene. The transcription of KCNQ10T1 is regulated by methylation at DMR2 (Fig. 1).

IGF2 is expressed exclusively from the paternal allele and is silent on the maternal allele. H19 has the opposite imprint, expressed only from the maternal allele and silent on the paternal allele (Fig. 1). DNA methylation plays a critical role in the establishment of the correct chromatin structure for the imprinted expression of these two genes. In part, formation of the correct chromatin structure is mediated by the CTCF chromatin insulator, which is sensitive to DNA methylation; thus,

\footnotetext{
From the ${ }^{I}$ Department of Human Genetics, Emory University School of Medicine, Atlanta, Georgia; ${ }^{2}$ Molecular Genetics Laboratory, Mayo Clinic, Rochester, Minnesota; ${ }^{3}$ Department of Genetics, La Paz University Hospital, Madrid, Spain.

Bradford Coffee, PhD, Department of Human Genetics, Emory University, 375 Whitehead Research Bldg., Atlanta, GA 30322.

Submitted for publication April 5, 2006
}

Accepted for publication June 27, 2006.

DOI: 10.1097/01.gim.0000237770.42442.cc
CTCF will only interact with unmethylated DNA. ${ }^{3}$ There are seven binding sites for CTCF, located between the IGF2 and $\mathrm{H} 19$ genes. In healthy individuals the CTCF sites are methylated on the paternal allele, along with the $H 19$ promoter, and unmethylated on the maternal allele. ${ }^{4}$

CTCF binding to its cognate sites on the unmethylated maternal allele allows the formation of a chromatin loop that results in the expression of $H 19$ and silences IGF2. ${ }^{5}$ Gaston et al. ${ }^{2}$ found that 58 of 97 patients (60\%) displayed hypomethylation of DMR2. Of the remaining 39 patients, 13 displayed hypermethylation of DMR1. Altogether, 71 of 97 patients (73\%) displayed hypomethylation of DMR2 or hypermethylation of DMR1. The hypermethylation of $H 19$ results in an increase in expression of IGF2 leading to the overgrowth phenotype. It is important to note that only methylation at the $\mathrm{H} 19$ promoter, and not at the CTCF binding sites, is assessed in clinical laboratory testing.

Recently, the opposite methylation defect was identified in patients diagnosed with Silver-Russell Syndrome (SRS). ${ }^{6}$ In some patients diagnosed with SRS there is hypomethylation of the CTCF binding sites causing a decrease in IGF2 expression, resulting in the growth retardation phenotype. In SRS hypomethylation of $\mathrm{H} 19$ is found in approximately $35 \%$ of patients clinically diagnosed with the disease. ${ }^{7}$

Defects in DNA methylation are also found at the centromeric DMR2, resulting in BWS. ${ }^{8}$ Unlike DMR1, defects in DNA methylation at DMR2 have not been associated with SRS. ${ }^{6}$ The most common DNA methylation abnormality 


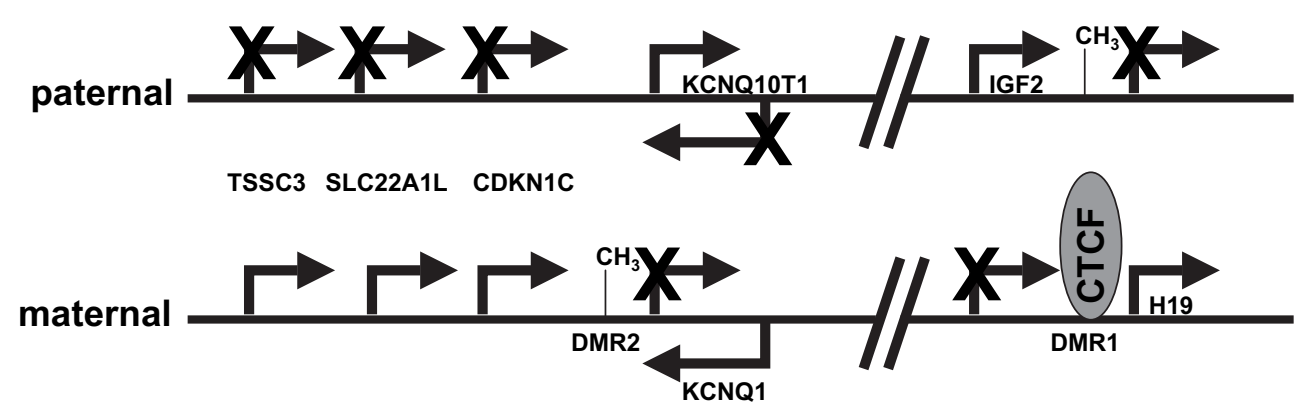

Centromeric domain Telomeric domain

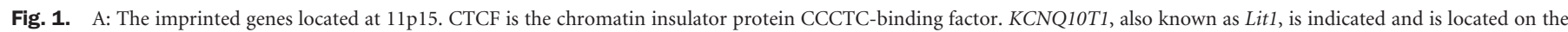

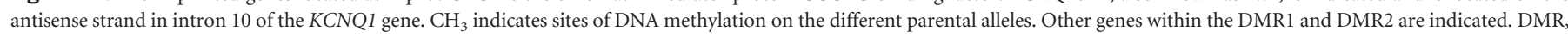
differentially methylated region.

found in BWS is hypomethylation of the KCNQ10T1 promoter, which results in the aberrant expression of the KCNQ10T1 antisense transcript leading to the silencing of the genes located within DMR2. Approximately $60 \%$ of the cases of BWS are associated with loss of KCNQ10T1 DNA methylation..$^{2,8}$ Paternal uniparental disomy is associated with approximately $10 \%$ to $20 \%$ cases, also resulting in loss of expression of the KCNQ1, CDKN1C, SLC22A1L, and TSSC $3 .{ }^{9}$ Therefore, in approximately $70 \%$ to $80 \%$ of BWS cases there is partial or complete loss of KCNQ10T1 DNA methylation, either because of hypomethylation of the maternal KCNQ10T1 allele or segmental paternal uniparental disomy for chromosome 11.

Quantitative methylation-sensitive polymerase chain reaction (PCR) assays, such as MethylLight, were developed as an alternative to Southern analysis to detect and quantify changes in DNA methylation in tumor-suppressor genes in cancer. ${ }^{10,11}$ In this method, genomic DNA is treated with sodium bisulfite, which deaminates unmethylated cytosines to uracil. Methylated cytosines are resistant to this deamination and after sodium bisulfite treatment will remain as cytosines. After desulfonation and purification the DNA is used as a template in a real-time TaqMan (Applied Biosystems, Foster City, CA) PCR reaction with amplification primers that are designed to avoid cytosines in $\mathrm{CpG}$ dinucleotides. This allows for the equivalent amplification of both methylated and unmethylated alleles. During PCR amplification different fluorescently labeled TaqMan probes are used to discriminate between methylated and unmethylated DNAs. By interpolation on standard curves, generated simply by titration of a sample from an unaffected individual, the amount of methylated and unmethylated DNA can be determined. A methylation index (MI), defined as the amount of methylated DNA divided by methylated plus and unmethylated DNA, can be calculated. Deviation from a predetermined normal range would be indicative of disease.

In clinical laboratory testing DNA methylation defects found in patients with BWS are routinely assessed by Southern analysis using methylation-sensitive restriction enzymes that target the promoters of $H 19$ (an SmaI site for DMR1 methylation analysis) or the promoter of the KCNQ10T1 gene (a NotI site for DMR2 methylation analysis). In the TaqMan MSP assay reported here we designed amplification primers and Taq-
Man probes that target the promoter of the KCNQ10T1 gene, immediately upstream of the transcription initiation site, and CTCF binding site 6 , located approximately $2 \mathrm{~kb}$ upstream of the SmaI site currently used for clinical laboratory testing. We compared the real-time TaqMan method with Southern analysis to determine the validity of this assay for clinical testing.

\section{MATERIALS AND METHODS}

\section{Sodium bisulfite treatment}

One microgram of genomic DNA from affected and unaffected individuals was diluted in $25 \mu \mathrm{L} \mathrm{dH_{2 }} \mathrm{O}$. The DNA was denatured by the addition of $2.75 \mu \mathrm{L}$ of $2 \mathrm{~N} \mathrm{NaOH}$, final concentration of $0.2 \mathrm{~N} \mathrm{NaOH}$, and incubated at $37^{\circ} \mathrm{C}$ for $10 \mathrm{~min}-$ utes. After denaturation, $15 \mu \mathrm{L}$ of freshly prepared $10 \mathrm{mM}$ hydroquinone (Sigma cat no. H 9003) and $260 \mu \mathrm{L}$ of $3.6 \mathrm{M}$ sodium bisulfite pH5.0 (Sigma cat no. 243973) were added, and the reaction was layered with mineral oil and incubated overnight ( 16 hours) at $54^{\circ} \mathrm{C}$. A modification of the protocol for the Wizard SV Genomic DNA Clean-Up System (Promega A2361; Madison, WI) was used to isolate the DNA after sodium bisulfite treatment. Briefly, $300 \mu \mathrm{L}$ of a 1:1 mix of SV lysis buffer and $95 \%$ ethanol was mixed with the $300 \mu \mathrm{L}$ of sodium bisulfite reaction. This mixture was transferred to a spin column and centrifuged 1 minute to bind the DNA to the resin within the column. The sample was washed two times with 600 $\mu \mathrm{L}$ of SV wash buffer, centrifuging 1 minute for each wash. The column was centrifuged one more time without wash buffer to remove residual buffer and transferred to a new $1.5-\mathrm{mL}$ microfuge tube. The DNA was eluted from the column by adding 50 $\mu \mathrm{L}$ of $\mathrm{H}_{2} \mathrm{O}$ followed by centrifugation for 1 minute. The DNA eluted from the column was desulfonated by the addition of 5.5 $\mu \mathrm{L} 3 \mathrm{~N} \mathrm{NaOH}$ and incubation at room temperature for 5 minutes. The DNA was ethanol precipitated, washed one time with $75 \%$ ethanol, and suspended in $50 \mu \mathrm{L}$ of $10 \mathrm{mM}$ Tris $\mathrm{HCl}$ (pH 8.0).

Real-time TaqMan methylation-sensitive polymerase chain reaction Primer and probe design for DMR2: The KCNQ10T1 promoter

All primers for the TaqMan MSP were synthesized by Integrated DNA Technologies (Coralville, IA). The amplification 
primers used in the real-time TaqMan methylation-sensitive PCR reaction were designed to avoid $\mathrm{CpG}$ dinucleotides in sense strand in the promoter of the KCNQ10T1 gene, which is antisense to the KCNQ1 gene. The KCNQ10T1 amplification primers are Lit1FGTTTAATTAGTAGGTGGGGGG and Lit1R-CCTAACAAAATCTTACTAAAAAACTCC. The TaqMan probes target the sequence -6 to -34 relative to transcription initiation of KCNQ10T1 (Genbank AJ006345; positions 255,041-255,069 on the antisense strand). The TaqMan probes sequences are Lit1-MCGGCGGGGGTAGTCGGAGCG and Lit1-U TGGTGGGGGTAGTTGGAGTGTTGTTGTA $\overline{\mathrm{G}}$ (underlined sequences indicate $\mathrm{CpG}$ dinucleotides used to discriminate between methylated and unmethylated DNA). The methylated DNA-specific probe is $5^{\prime}$ end-labeled with FAM and quenched by the addition of Black Hole Quencher (Biosearch Technologies Inc., Novato, CA) 1 to the $3^{\prime}$ end of the oligonucleotide. The unmethylated DNA specific probe was $5^{\prime}$ end-labeled with HEX and quenched by the addition of Black Hole Quencher 1 to the 3' end of the oligonucleotide.

\section{Primer and probe design for DMR1-CTCF binding site 6}

The amplification primers target the sense strand and flank the CTCF binding site 6 , located upstream of the $H 19$ gene. The CTCF-6 amplification primers were CTCF6F-GTATAGTATATGGGTATTTTTGGAGG and CTCF6R-CCCAATTAAAACRAACTCRAACTATAAT. The TaqMan probes target the core sequence of the CTCF binding site 6 (Genbank AC087017; positions 6183-6206). The probe sequences were CTCF6M- AAGTGGTCGCGCGGCGGTAGTGTA and CTCF6U-TGGAAGTGGTTGTGTGGTGGTAGTGTAGG. As with the KCNQ10T1 promoter, the TaqMan probe specific for the methylated DNA is labeled with FAM and the unmethylated DNA specific probe is labeled with HEX. Both probes were quenched by the addition of Black Hole Quencher 1 to the $3^{\prime}$ end of the oligonucleotide.

\section{Real-time TaqMan methylation-sensitive polymerase chain reaction set-up}

In separate reactions the methylation status of the KCNQ10T1 promoter and CTCF binding site 6 were assessed using $16 \mathrm{ng}$ of sodium bisulfite-treated genomic DNA suspended in $5 \mu \mathrm{L}$ of $10 \mathrm{mM}$ Tris $\mathrm{HCl}(\mathrm{pH}$ 8.0). The PCR was performed in a $25 \mu \mathrm{L}$ reaction with $1 \times$ Invitrogen (Carlsbad, CA) PCR buffer (20 mM Tris- $\mathrm{HCl} \mathrm{pH} 8.4$ and $50 \mathrm{mM} \mathrm{KCl}), 1$ $\mathrm{mM} \mathrm{MgCl} 2,250 \mu \mathrm{M} \mathrm{dNTP}, 2.5 \mathrm{pmol} / \mu \mathrm{L}$ of each amplification primer and $0.15 \mathrm{pmol} / \mu \mathrm{L}$ of each TaqMan probe, and 8 units of Invitrogen Platinum Taq (catalog no. 10966). The PCR cycling parameters using a BioRad iQ5 Light-Cycler (Hercules, CA) were initial denaturation at $95^{\circ} \mathrm{C}$ for 3 minutes followed by $95^{\circ} \mathrm{C}$ for 10 seconds, $52^{\circ} \mathrm{C}$ for 30 seconds, and $72^{\circ} \mathrm{C}$ for 30 seconds, repeated 40 times. In parallel with each set of reactions a standard curve was generated using a sodium bisulfite-treated genomic DNA sample from an unaffected individual. The range of DNA amount used in the standard curve was from 1 to $64 \mathrm{ng}$.

\section{Data analysis}

The amount of methylated and unmethylated DNA was calculated for each sample, which was assayed in triplicate, by interpolation on the standard curve that was run in parallel with the unknown samples. The MI was determined by dividing the amount of methylated DNA by the amount of total DNA (methylated plus unmethylated DNA). The mean of the three assays was used to calculate the MI for that patient. A normal range was generated by testing a series of unaffected individuals, whom were also assessed in triplicate. The mean and standard deviation (SD) were calculated for this set of samples, and as with the Southern method, ${ }^{2}$ the normal range was defined as the mean $\pm 2 \mathrm{SD}$.

\section{RESULTS}

\section{Assessment of DNA methylation of DMR2 in healthy individuals}

Initially, we chose to develop an assay for BWS that would have the highest yield, accounting for $60 \%$ to $80 \%$ of cases, methylation analysis of DMR2, the KCNQ10T1 promoter. The amplification primers that target the sense strand of DMR2 were designed to avoid $\mathrm{CpG}$ dinucleotides, eliminating any bias in amplification between methylated and unmethylated templates. The TaqMan probes target the KCNQ10T1 promoter at positions -15 to -34 for the methylated DNA-specific probe and positions -6 to -34 for the unmethylated DNA-specific probe relative to the transcription initiation site. ${ }^{12}$ The probes were labeled with FAM for methylated DNA and with HEX for unmethylated DNA.

We generated a standard curve by titrating sodium bisulfitetreated DNA, from 1 to $64 \mathrm{ng}$, from an unaffected individual. The curve was then used to determine the values of the unknown samples. A representative example of the amplification plots and the resulting standard curves for both methylated DMR2 DNA and unmethylated DMR2 DNA are shown in Figure 2. The crossing threshold for each individual amplification correlates with the amount of input DNA allowing the generation of standard curves with correlation coefficients $\left(\mathrm{R}^{2}\right)$ consistently greater than 0.95 .

\section{DMR2 DNA methylation in patients with Beckwith-Wiedemann Syndrome}

We tested a series of unaffected individuals to establish a normal range of DNA methylation detected in the assay (Fig. $3 \mathrm{~A})$. In Southern analysis for BWS the normal range is defined as the mean $\pm 2 \mathrm{SD}$. For clinical testing by Southern analysis the normal range is accepted to be 0.40 to $0.60 .^{2} \mathrm{An}$ individual with an MI 2 SD below that mean is considered to have loss of methylation of DMR2, consistent with a diagnosis of BWS. In Figure 3A, we show the MIs for 15 unaffected individuals assessed for DNA methylation changes at DMR2. Eight of these individuals were also assessed by Southern analysis, shown as the red bars for samples 1 to 8 . In total, 92 DNA samples from individuals not diagnosed with BWS were assessed for methylation at DMR2. The average MI was 0.52 with an SD of 0.06 (data not shown).

We also assessed DMR2 methylation in a series of patients with BWS who previously were identified to have loss of methylation by Southern analysis. In Figure 3B, we compare the MIs 

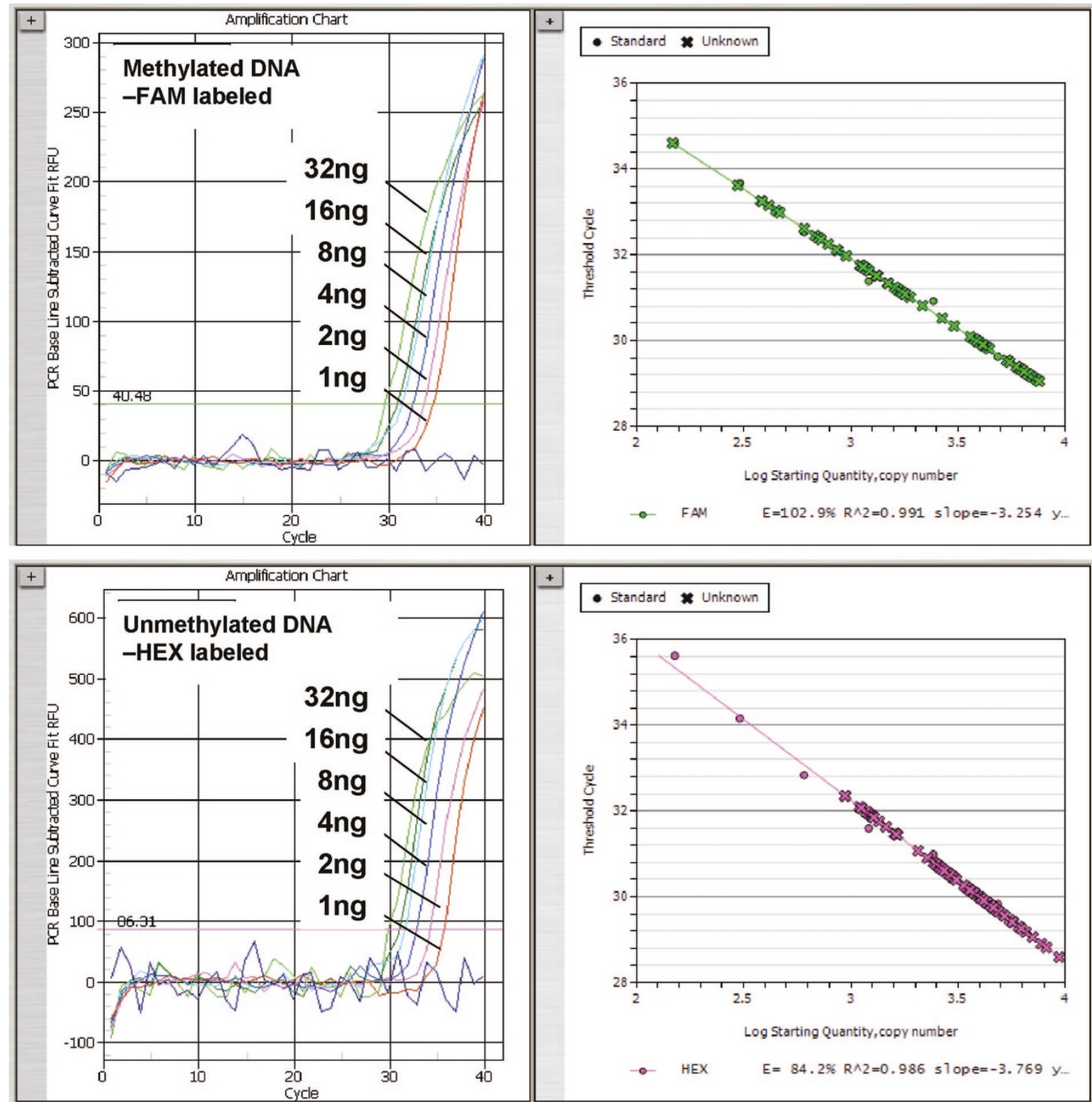

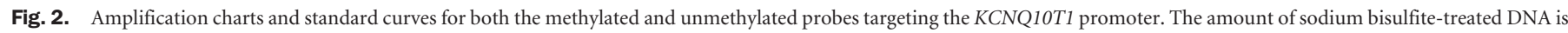

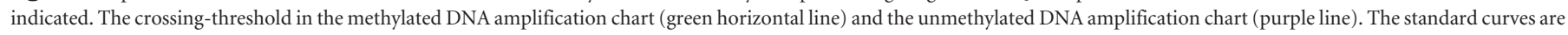
plotted to the right of each amplification chart. The known standards (filled circles) and the unknown samples (X's).

from TaqMan MSP versus Southern analysis in 14 patients with BWS. In all of the patients with complete (or near complete) loss of DMR2 methylation (samples 1-7), as well as in samples with partial loss of DNA methylation (samples 8-14), the TaqMan MSP detected loss of KCNQ10T1 methylation that was similar to the MIs determined by Southern analysis. All of the TaqMan MSP analyses were performed blinded to knowledge of the Southern analysis results.

\section{DNA methylation of CTCF binding site 6 upstream of $\mathbf{H 1 9}$}

One advantage of TaqMan MSP is that primers and probes can be designed to assess DNA methylation at any site in the genome. Because alterations in DNA methylation in DMR1 are also implicated in BWS and SRS, we designed primers to assess DNA methylation at this locus as well. Approximately $33 \%$ of patients with BWS who test negative for loss of KCNQ10T1 methylation have hypermethylation of the $H 19$ promoter. $^{2}$ In addition, approximately $35 \%$ of patients clinically diagnosed with SRS have hypomethylation at H19.7 Instead of targeting the H19 promoter for methylation analysis, we designed TaqMan probes and amplification primers that directly target the CTCF binding site 6 . This site is located at positions -2123 to -2137 relative to the $H 19$ gene transcription start site. In the Southern analysis used in clinical testing, an SmaI site (located 

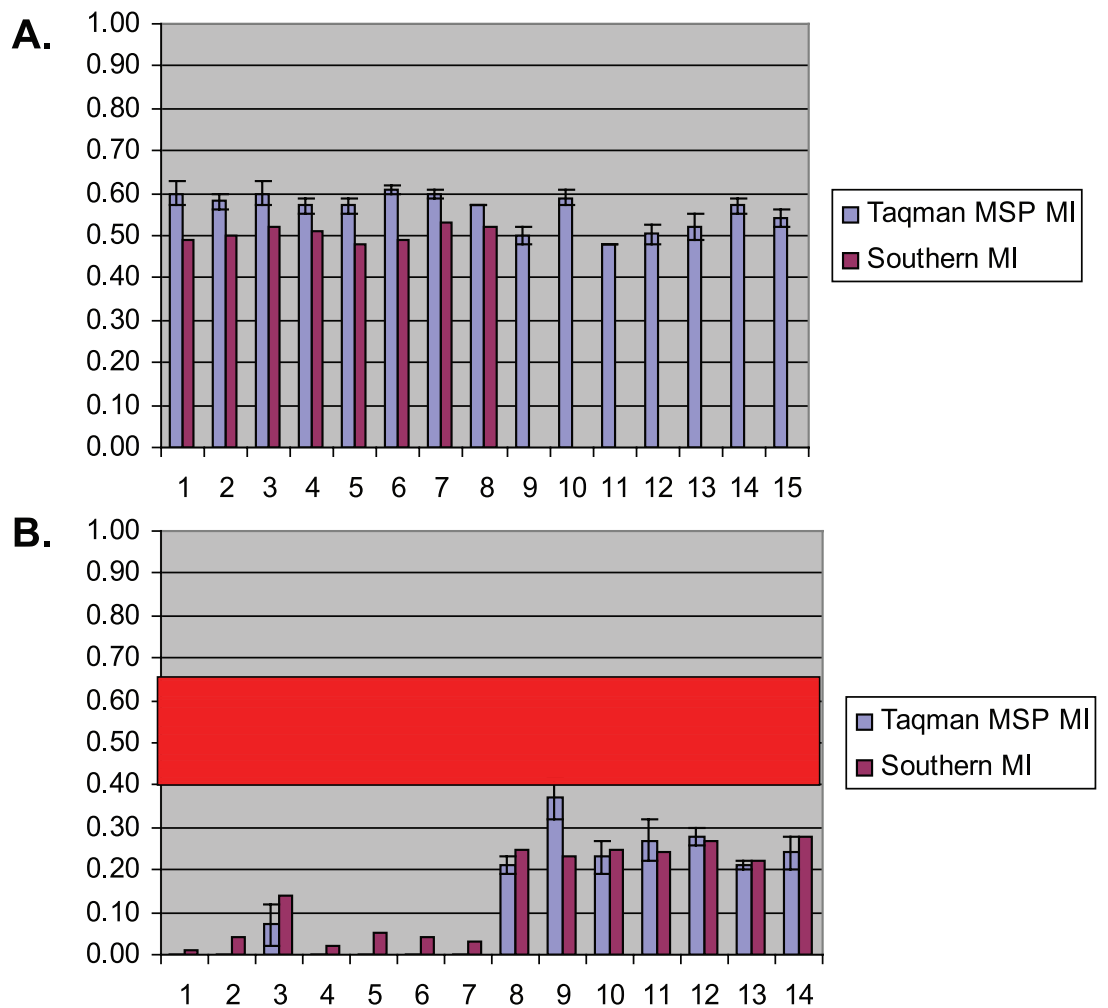

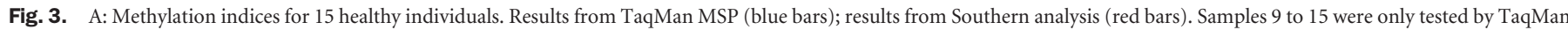

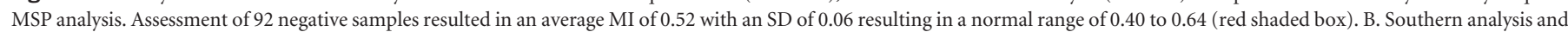

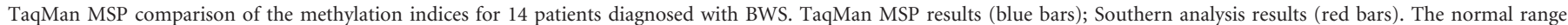
(horizontal red box) determined in Figure 3A. MI, methylation index.

at position -15 relative to transcription initiation) is targeted for DNA methylation analysis. ${ }^{2}$

In Figure 4A we test a series of 11 controls to establish a normal range for the MI at DMR1. The mean from these 11 controls was 0.52 with an SD of 0.05, resulting in a normal range from 0.42 to 0.62 . This compares favorably with a mean of 0.51 and a normal range of 0.43 to 0.59 for Southern analysis (P. Lapunzina, MD, unpublished data, 2006). In total, 91 DNA samples from healthy individuals were assessed for methylation at DMR1. The average MI was 0.49 with an SD of 0.08 (data not shown).

In addition, we tested 27 patients with BWS who were previously tested by Southern analysis for methylation defects at DMR1 and DMR2 by TaqMan MSP. All of the TaqMan MSP results for DMR2 methylation analysis were completely concordant with the Southern analysis results (data not shown). The DMR1 analysis results are shown in Figure 4B. As with DMR2 methylation analysis, we were blinded to the results of the DMR1 Southern analysis.

Of the five patients who scored positive for hypermethylation at CTCF6 (MI > 0.65), all five scored positive for H19 promoter hypermethylation by Southern analysis (MI >0.60), demonstrating the concordance between the two methods. One patient, sample 7, had a high normal TaqMan MSP MI of 0.62 and a Southern MI of 0.65. This patient, therefore, had a TaqMan MI slightly below the cutoff of 0.65 , but scored positive by Southern analysis with an MI slightly above the cutoff of
0.60. This patient also had hypomethylation of DMR2 both by TaqMan MSP and Southern analysis (data not shown) and has a duplication of $11 \mathrm{p} 15 .{ }^{13}$

\section{Determining the parent of origin of a cytogenetically identified 11 15 duplication}

We identified a duplication of $11 \mathrm{p} 15$ by chromosome analysis in an infant with some of the clinical features of BWS, but without a clear clinical diagnosis of the disease. We used the combined assay of DMR1 and DMR2 methylation analysis to determine the parent of origin of this duplicated chromosome $11 \mathrm{p} 15$ segment. If the duplication was paternal in origin, as is found in some cases of BWS, we would expect a decrease in the MI for DMR2 from 0.5 to 0.33 because there would be two unmethylated paternal copies of DMR2 for every methylated maternal copy of DMR2. At the same time we would see an increase in the MI at DMR1 from 0.5 to 0.66 . In this case there would be two methylated paternal copies of the DMR1 for every unmethylated maternal copy of DMR1. As seen in Figure 5 , there is a shift in the MIs predicted for a paternal origin of this duplication indicating that the child has a methylation profile consistent with a diagnosis of BWS.

\section{DISCUSSION}

We assayed a series of controls and patients diagnosed with BWS at two different loci at $11 \mathrm{p} 15$ to assess the validity of the 

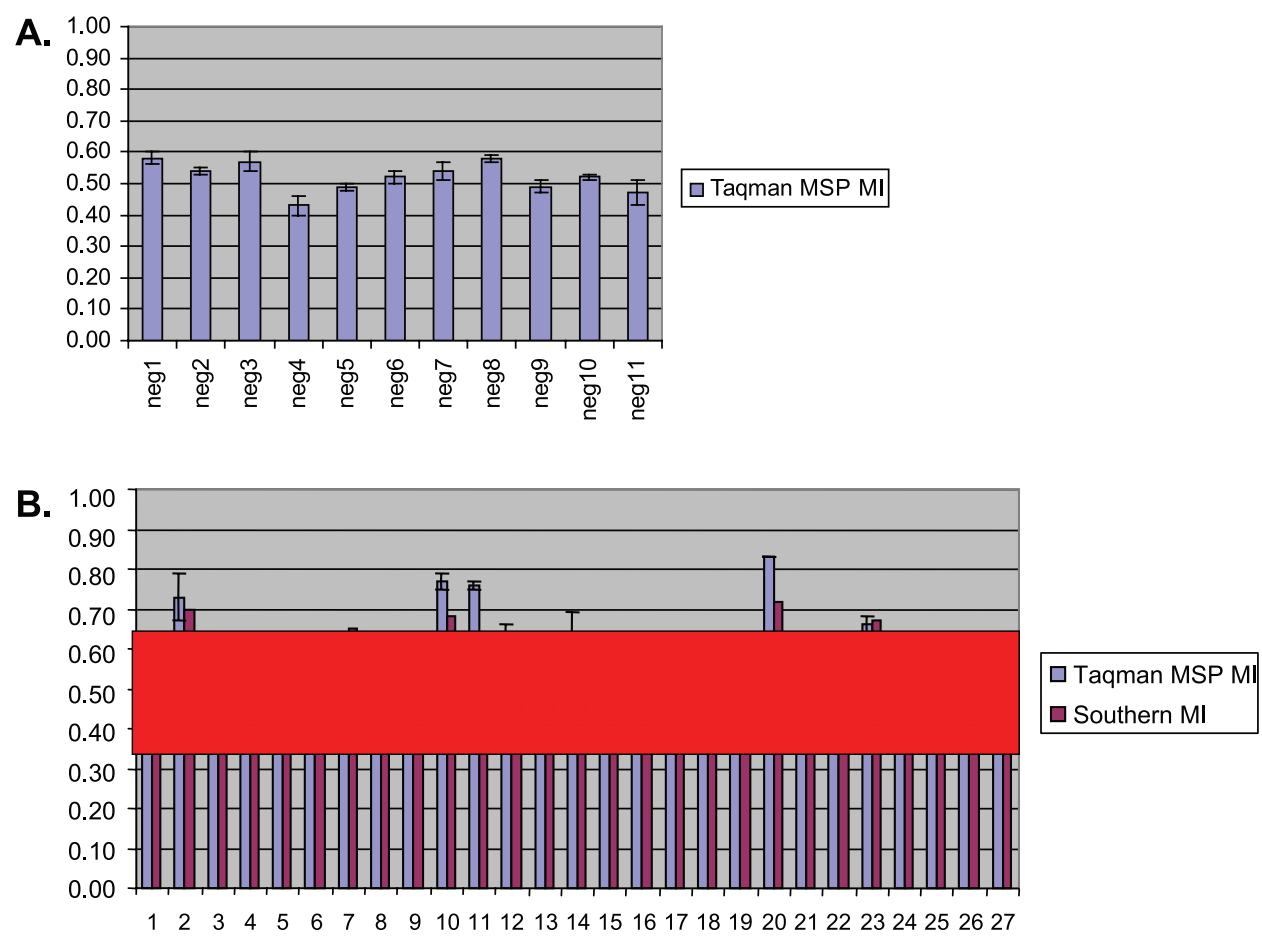

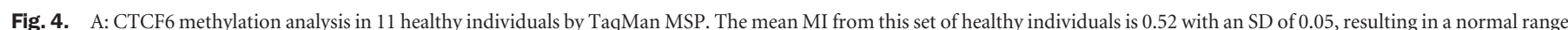

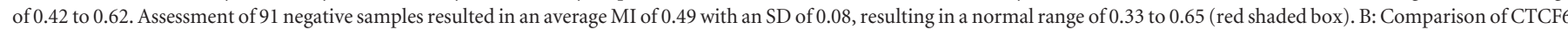

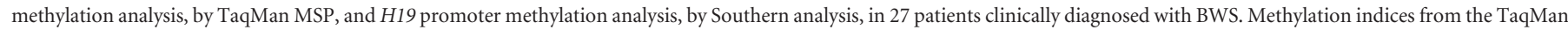
analysis (blue bars); methylation indices from Southern analysis (red bars). The normal range (horizontal red box) from Figure 4A. MI, methylation index.

TaqMan MSP method for clinical laboratory testing. These results compare favorably, at both DMR1 and DMR2, with Southern blot analysis. Simultaneous assessment of both DMR1 and DMR2 by TaqMan MSP provides the most comprehensive assay for methylation defects in BWS, accounting for more than $70 \%$ of the cases. ${ }^{2}$

Loss of $\mathrm{H} 19$ methylation has recently been found in patients clinically diagnosed with SRS. ${ }^{6}$ TaqMan MSP would also be able to test for $H 19$ hypomethylation in these individuals. In their investigation, Gicquel et al. ${ }^{6}$ specifically targeted CTCF binding site 6 by sodium bisulfite DNA sequencing analysis in two patients who also displayed loss of methylation at the $\mathrm{H} 19$ promoter. Quantification of the methylation at CTCF binding site 6 showed a more pronounced hypomethylation than that found at the $H 19$ promoter (patient 3 had a $29.2 \% \mathrm{MI}$ at $H 19$ vs. $21 \% \mathrm{MI}$ at CTCF binding site 6; patient 8 had $8.5 \% \mathrm{MI}$ at $\mathrm{H} 19$ vs. $4.8 \%$ at CTCF6). This observation is consistent with the idea that CTCF binding site 6 may be a better target for methylation analysis than the $\mathrm{H} 19$ promoter. It is estimated that approximately $35 \%$ of patients clinically diagnosed with SRS have hypomethylation of DMR1.7

There are several advantages of the TaqMan MSP over Southern analysis. Only $1 \mu \mathrm{g}$ of DNA is required for the TaqMan MSP method as opposed to up to $10 \mu \mathrm{g}$ required for

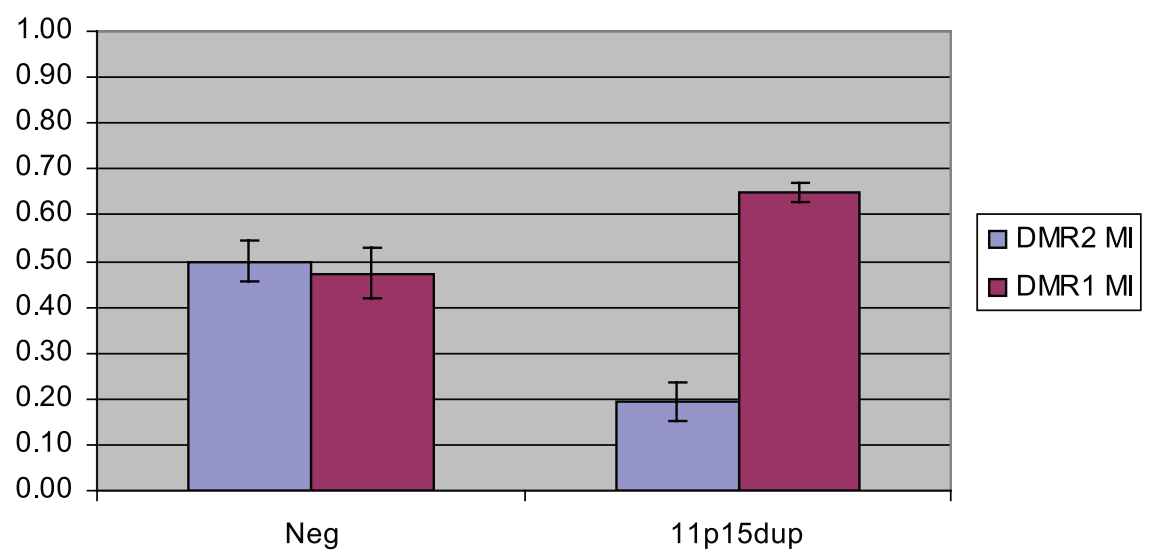

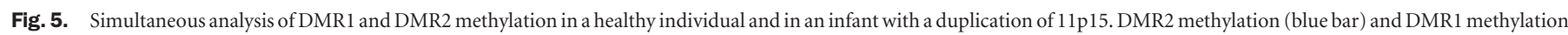
(red bar). MI, methylation index. 
Southern analysis. A second advantage is that the assay can be completed in 2 days versus the typical 1 week required for Southern analysis, resulting in faster turnaround time. A third advantage is that the TaqMan MSP is less labor-intensive and therefore more cost-effective. A fourth advantage is that the assay is amenable to high-throughput analyses. For example, with a 96-well format instrument such as the iQ5 iCycler (BioRad), 28 samples can be assayed simultaneously in triplicate, along with the controls for generating the standard curve. Finally, with this method any site in the genome can be targeted for DNA methylation analysis. By simply designing amplification primers that avoid $\mathrm{CpG}$ dinucleotides and TaqMan probes that discriminate between methylated and unmethylated DNA after sodium bisulfite conversion, any $\mathrm{CpG}$ island in the human genome can be assessed for alterations in DNA methylation. Thus, clinical laboratory testing using this approach could be readily developed for all human epigenetic disorders.

\section{References}

1. Weksberg R, Smith AC, Squire J, Sadowski P. Beckwith-Wiedemann syndrome demonstrates a role for epigenetic control of normal development. Hum Mol Genet 2003;12:R61-R68.

2. Gaston V, Le Bouc Y, Soupre V, Burglen L, et al. Analysis of the methylation status of the KCNQ1OT and H19 genes in leukocyte DNA for the diagnosis and prognosis of Beckwith-Wiedemann syndrome. Eur J Hum Genet 2001;6:409-418.
3. Bell AC, Felsenfeld G. Methylation of a CTCF-dependent boundary controls imprinted expression of the IGF2 gene. Nature 2000;405:482-485.

4. Sparago A, Cerrato F, Vernucci M, Ferrero GB. et al Microdeletions in the human H19 DMR result in loss of IGF2 imprinting and Beckwith-Wiedemann syndrome. Nat Genet 2004;36:958-960.

5. Murrell A, Heeson S, Reik W. Interaction between differentially methylated regions partitions the imprinted genes IGF2 and H19 into parent-specific chromatin loops. Nat Genet 2004;36:889-893.

6. Gicquel C, Rossignol S, Cabrol S, Houang M, et al. Epimutation of the telomeric imprinting center region on chromosome 11 p15 in Silver-Russell syndrome. Nat Genet 2005;37:1003-1007.

7. Eggermann T, Schonherr N, Meyer E, Obermann C, et al. Epigenetic mutations in 11 p15 in Silver-Russell syndrome are restricted to the telomeric imprinting domain. J Med Genet 2006;43:615-616. Epub 2005 Oct 19.

8. Smilinich NJ, Day CD, Fitzpatrick GV, Caldwell GM, et al. A maternally methylated CpG island in KvLQT1 is associated with an antisense paternal transcript and loss of imprinting in Beckwith-Wiedemann syndrome. Proc Natl Acad Sci U S A 1999;96: 8064-8069.

9. Henry I, Bonaiti-Pellie C, Chehensse V, Beldjord C, et al. Uniparental paternal disomy in a genetic cancer-predisposing syndrome. Nature 1991;351:665-667.

10. Eads CA, Danenberg KD, Kawakami K, Saltz LB, et al. MethyLight: a high-throughput assay to measure DNA methylation. Nucleic Acids Res 2000;28:E32.

11. Zeschnigk M, Bohringer S, Price EA, Onadim Z, et al. A novel real-time PCR assay for quantitative analysis of methylated alleles (QAMA): analysis of the retinoblastoma locus. Nucleic Acids Res 2004;32:e125.

12. Du M, Zhou W, Beatty LG, Weksberg R, et al. The KCNQ1OT1 promoter, a key regulator of genomic imprinting in human chromosome 11p15.5. Genomics 2004; 84:288-300.

13. Delicado A, Lapunzina P, Palomares M, Molina MA, et al. Beckwith-Wiedemann syndrome due to $11 \mathrm{p} 15.5$ paternal duplication associated with Klinefelter syndrome and a "de novo" pericentric inversion of chromosome Y. Eur J Med Genet 2005;48: $159-166$. 